\title{
True Angle between Anterior-Posterior Impingement in Total Hip Arthroplasty: Why Common Constrained Cups have to Fail
}

Andrej M. Nowakowski ${ }^{1^{*}}$, Sebastian Gehmert ${ }^{1^{*}}$, Irène Roesle ${ }^{1}$ and Karl-Heinz Widmer ${ }^{2}$

${ }^{1}$ Orthopaedic Department, University of Basel, Spitalstrasse 21, 4031 Basel, Switzerland

2Orthopaedic Department, Kantonsspital Schaffhausen, Geissbergstrasse 81, 8208 Schaffhausen, Switzerland

*Corresponding author: Andrej M. Nowakowski, Orthopaedic Department, University of Basel, Spitalstrasse 21, 4031 Basel, Switzerland, Tel: +41-61 26 57197; E-mail: andrej.nowakowski@unibas.ch

Sebastian Gehmert, Orthopaedic Department, University of Basel, Spitalstrasse 21, 4031 Basel, Switzerland, E-mail: sebastian.gehmert@usb.ch

Received: May 07, 2016; Accepted: May 20, 2016; Published: May 24, 2016

Copyright: ( 2016 Gehmert G, et al. This is an open-access article distributed under the terms of the Creative Commons Attribution License, which permits unrestricted use, distribution, and reproduction in any medium, provided the original author and source are credited.

\begin{abstract}
Background: Dislocation after total hip arthroplasty is a common complication and partially based on impingement.
\end{abstract}

Questions/purposes: (1) The purpose of this study was to investigate the angle between anterior and posterior impingement in current hip cup designs. (2) Furthermore, the aim was to design a model of an acetabular cup with sparing gaps that match to impact areas.

Methods: The range of motion was simulated with Maple R8 software using standard parameters in hip arthroplasty. Afterwards, a preliminary model for an optimized acetabular cup was designed in order to avoid impingement and dislocation.

Results: (1) Anterior and posterior areas of impingement were not opposite but twisted by an angle of $108.3^{\circ}$. (2) The two main trajectories of motion were identified and areas with corresponding reductions and elevations were appropriate modified. The improvement resulted in a "bidirectional total hip prosthesis" with a combination of a snapfit acetabular cup and a reduced cup profile.

Conclusion: The improvements of the described hip prosthesis are based on a simulation and are most likely to prevent impingement and subsequent dislocation. In addition, simulation with standard implantation parameters resulted in a rotational asymmetric implant design.

Clinical relevance: Our data provide evidence that conventional hip cup designs fail to prevent impingement due to (1) The incorrect assumption of diagonally arranged impingement areas and the diagonal arranged sparing gaps (2) The sparing gap design itself that technically is not reducing the rim of the cup but instead only has an elevated coverage relative to the center of motion.

Keywords: Total Hip Arthroplasty; Dislocation; Impingement; Constrained cup; Bidirectional

\section{Introduction}

Dislocation remains a major complication after total hip replacement (THR) that may require challenging revision surgery [1]. The incidence of dislocation after primary hip replacement is reported with a range between $0.4 \%$ and $7 \%$ [2-6]. In addition, the risk for dislocation is markedly increased up to $15 \%$ after a revision of a hip prosthesis [6].

Problems caused by dislocation after primary THR are frequently underestimated owing to the relative low incidence compared to the general long life cycle of primary implants. Noteworthy, impingement plays a crucial role for a dislocation after primary THR with almost one quarter of all revisions [7]. Impingement appears when the postoperative range of motion (RoM) exceeds the technical limits of the implant in at least one motion axis [1]. Moreover, a poor alignment of the components during implantation can further decrease the RoM resulting in increased impingements [1]. The prosthetic neck can be stressed by hitting the liner or cup. Consequently, the head center will translate relative to the cup or liner since the neck acts as a lever at the moment of impact. Head dislocation occurs if the translation of the head centre exceeds a critical magnitude.

It is noteworthy that evidence of impingement was substantial present on the explanted acetabular cup in $56 \%$ of revisions to primary THR. This number increased to $94 \%$ when revision acetabular cups were examined on retrieval [8]. In line, Marchetti et al. [9] reported certain characteristics of impingement in $51.4 \%$ of 416 cups after dislocation. Noteworthy, Yamaguchi et al. reported a mean angle of $78^{\circ}$ between the posterior impingement site and the cephalic point in the acetabulum which was consistently apparent in all studied cases [10]. Interestingly, the authors also found anterior impingement but only in 
Citation: Nowakowski AM, Gehmert S, Roesle I, Widmer KH (2016) True Angle between Anterior-Posterior Impingement in Total Hip Arthroplasty: Why Common Constrained Cups have to Fail. Global J Technol Optim 7: 194. doi:10.4172/2229-8711.1000194

Page 2 of 5

3 cups and without stating the angle in association to the posterior impingement.

The concept of the bidirectional total hip prosthesis was introduced several years ago with a reconfigured wall design considering anticipated impingement areas [1]. The anterior and posterior margins are provided with sparing gaps whereas the medial and lateral portion of the acetabular component has an elevated coverage since the physiological hip RoM is distinctly greater in the flexion/extension than in the abduction/adduction level (Figure 1). Interestingly, the anterior and posterior sparing gaps are opposite to each other assuming that the anterior and posterior impingement occurs at an angle of $180^{\circ}$. However, to date no data exist regarding the exact angle between the anterior and posterior impingement in the coronal cup plane.

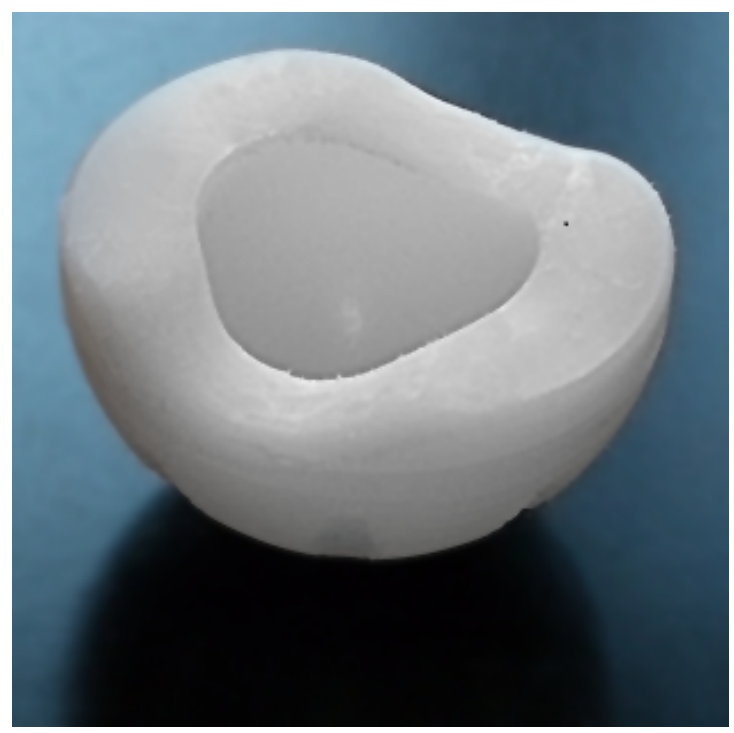

Figure 1: A simple model of a bidirectional total hip prosthesis. Anterior and posterior countersunk sparing along a snap fit cup with analogous regions of medial and lateral elevations. The angle of twist (AoT) is $180^{\circ}$ since the anterior and posterior sparing gaps are opposite to each other which assume that the anterior and posterior impingement occurs at an angle of $180^{\circ}$.

Based on our own experience with revision of acetabular components we hypothesized that the anterior and posterior impingement of the prosthetic neck to the acetabular component appears not opposite to each other with an angle of $180^{\circ}$. Thus, the purpose of this study was to investigate the true angle between anterior and posterior impingement in order to elucidate the mismatch between current hip cup design and mechanical requirements based on a range of motion analysis. Moreover, we intended to design a model for an improved acetabular cup with an exact match of sparing gaps and impingement areas based on simulated data in order to prevent impingement and required revision surgery.

\section{Materials and Methods}

For the RoM analysis, a 3-dimensional computerized model of a ball and socket joint was generated using Maple R8 software (Waterloo Maple Inc., Waterloo, Ontario, Canada) according to the recommendations of Widmer and Zurfluh [11-13]. The model was used to simulate any hip joint movement and to determine RoM for isolated or combined movements as previously described [13].

The following design-dependent standard parameters were fixed according to those reported in the analysis of optimal positioning by Widmer and Zurfluh [13], with measurements of the established total hip prostheses defined as:

\section{Cup}

Exterior Cup Diameter: $54 \mathrm{~mm}$

Articular Diameter: $28.2 \mathrm{~mm}$

\section{Head/Neck}

Head Diameter: $28 \mathrm{~mm}$

Neck Diameter: $12 \mathrm{~mm}$

Head/Neck Ratio: 2.33

\section{Stem}

Caput-collum-diaphyseal (CCD) angle: $130^{\circ}$

For adjustment of the implant-dependent parameters, the following optimal parameters for anteversion, inclination, and antetorsion were selected [13]:

\section{Cup}

Anteversion: $25^{\circ}$

Inclination: $44^{\circ}$

\section{Shaft}

\section{Antetorsion: $15^{\circ}$}

During RoM analysis, the center of the femoral head remained in the center of the articular surface. The neutral position of the femur was defined as $3^{\circ}$ of anterior flexion and $5^{\circ}$ of adduction [13].

Impact points of the neck on the cup were detected and displayed three dimensionally as points of impingement during the simulation of flexion-extension leg movements in the sagittal plane. In addition, we introduce a new angle of incline between anterior and posterior impingement area denominated as angle of twist (AoT) to underline the real angle association and to simplify further research communication.

Furthermore, the impingement points obtained from flexionextension simulation were used to generate a CAD model of an asymmetrical bidirectional total hip prosthesis. Finally, the virtual generated prosthesis was applied to a CT $3 \mathrm{D}$ pelvic reconstruction.

\section{Results}

The simulation of impingement using standard parameters revealed areas located anterior and posterior to the coronal cup plane axis (Figure 2A). However, the impingement areas were not located exact in the sagittal plane. Moreover, areas did not face each other with an AoT of $180^{\circ}$ (Figure $2 \mathrm{~B}$ ). Interestingly, the anterior and posterior impact points were positioned to each other with an AoT of $108.3^{\circ}$. In addition, the anterior impact point was identified at $32.2^{\circ}$ anterior to the coronal plane axis (perpendicular to the sagittal axis), whereas the posterior impact point was present at an angle of $76.1^{\circ}$ (Figure $2 \mathrm{~B}$ ). 
Citation: Nowakowski AM, Gehmert S, Roesle I, Widmer KH (2016) True Angle between Anterior-Posterior Impingement in Total Hip Arthroplasty: Why Common Constrained Cups have to Fail. Global J Technol Optim 7: 194. doi:10.4172/2229-8711.1000194

Page 3 of 5

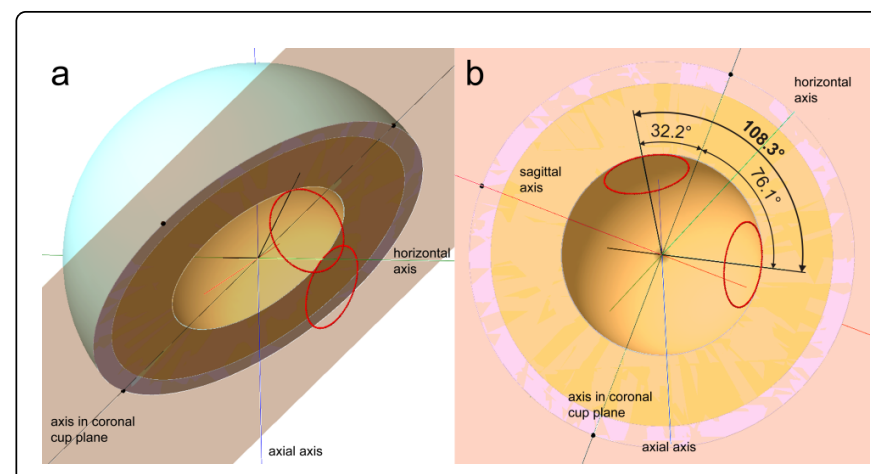

Figure 2: Representative graphical presentation of a RoM analysis result. Red circles designate the neck position at impingement points for A) a standard AP view of the acetabular cup and B) a view of the coronal cup plane using standard implantation parameters. The anterior and posterior impact points are positioned to each other with an AoT of $108.3^{\circ}$. The anterior impact point is located at $32.2^{\circ}$ anterior to the coronal plane axis (perpendicular to the sagittal axis), whereas the posterior impact point is present at an angle of $76.1^{\circ}$.

An additional simulation with incremental inclination of the cup showed that the impingement points moved towards each other with increasing inclination. At an inclination of $70^{\circ}$, anterior and posterior impingement areas were approximately perpendicular to each other $\left(\mathrm{AoT}=90^{\circ}\right)$. Collision between neck and cup $\left(\mathrm{AoT}=0^{\circ}\right)$ were prevented with a cup inclination of $80^{\circ}$. In addition, we defined the center of motion $(\mathrm{CoM})$ as the rotation angle bisecting the exterior AoT in the coronal cup plane. The center of motion appeared to be at $22.0^{\circ}$ when using the specified implant parameters. Finally, we were able to construct a model of an asymmetrical bidirectional total hip prosthesis based on our findings with reduced impingement areas (Figures 3 and 4).

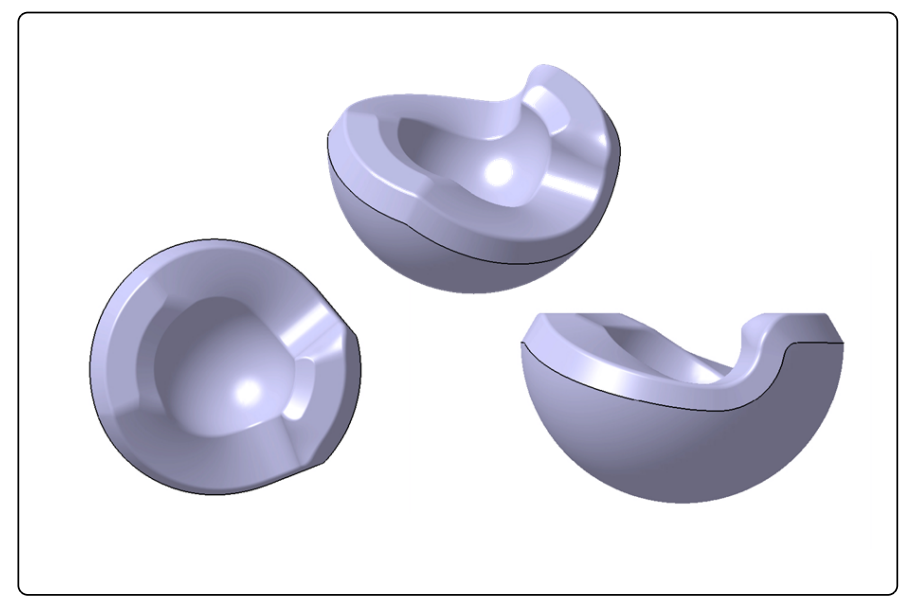

Figure 3: A simple CAD model of an asymmetrical bidirectional total hip prosthesis was constructed based on our findings of a true AoT of $108 \cdot 3^{\circ}$. A maximal reducing and elevation of $5 \mathrm{~mm}$ relating to the hemisphere was selected for the simulated CAD model.

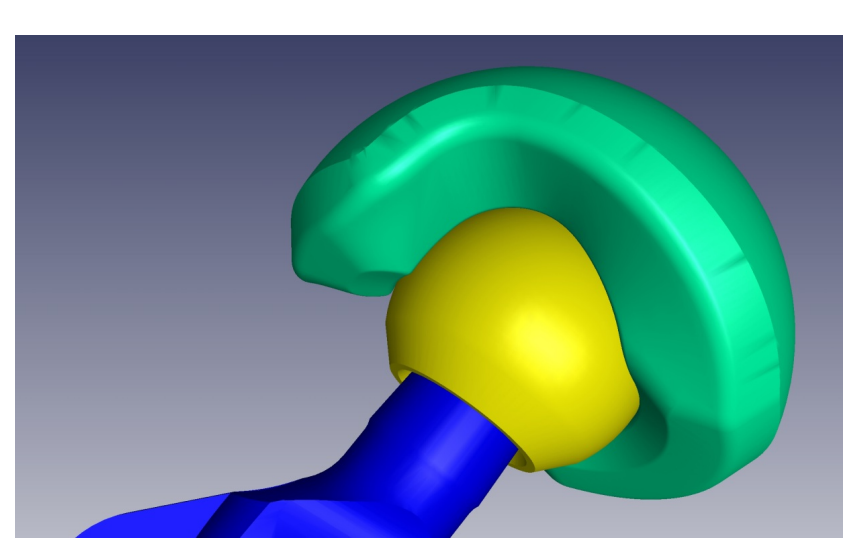

Figure 4: An extended simulation of an asymmetrical bidirectional total hip prosthesis (Figure 3) combined with a standard head (28 $\mathrm{mm}$ ) and a standard stem (Biocontact ${ }^{\circ}$, Aesculap AG, Tuttlingen, Germany) to demonstrate the 3 dimensional orientations of elevation and sparing gaps.

The virtual implantation into a CT $3 \mathrm{D}$ pelvic reconstruction revealed that the software-constructed prosthesis perfectly matches with anatomic features of the acetabulum since anterior sparing of the cup superposed to physiologic acetabulum sparing (Figure 5).

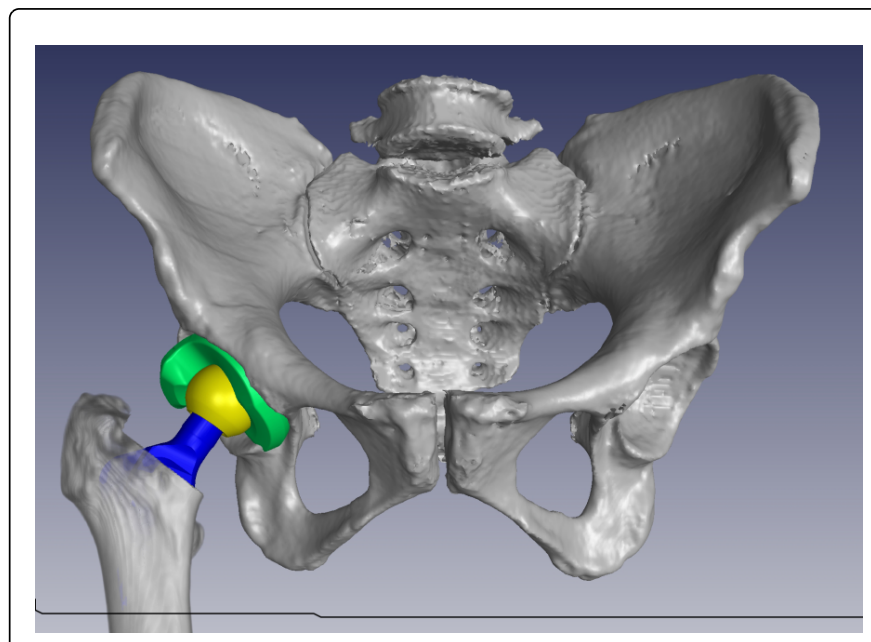

Figure 5: A virtual implantation of an asymmetrical bidirectional total hip prosthesis (Figure 4) to a 3D CT-Scan of a human pelvis. The anterior rim of the cup is shaped in a similar manner as the natural acetabular limbus.

\section{Discussion}

Dislocation and the consequences of impingement along with aseptic implant loosening are the most frequent mechanical factors for hip revision arthroplasty [1]. Previous studies provide evidence that posterior impingement is consistently apparent in the hip cup when revision surgery is needed. Current hip design solutions have assumed that the anterior and posterior impingement points using standard implant parameters are located opposite to each other with an approximate AoT of $180^{\circ}$. However, no data exist regarding the exact angle between the anterior and posterior impingement in the coronal cup plane. 
Citation: Nowakowski AM, Gehmert S, Roesle I, Widmer KH (2016) True Angle between Anterior-Posterior Impingement in Total Hip Arthroplasty: Why Common Constrained Cups have to Fail. Global J Technol Optim 7: 194. doi:10.4172/2229-8711.1000194

Page 4 of 5

Our study had some limitation. First, the present data were obtained by a simulated RoM analysis using software. The present study refers only to the selected implantation parameters and considers only an idealized extension/flexion motion in the sagittal plane without rotational components. Thus, the AoT value calculated here should not be over-interpreted as an actual individual value. In addition, the rotational asymmetry design based on the simulated RoM analysis requires a rotation in the coronal cup plane during the cup implantation.

We for the first time show, that the AoT with standard implant parameters is $108.3^{\circ}$ and far from the anticipated angle of $180^{\circ}$. Moreover, in the present study we also could show that the anterior and posterior impingement areas were approximately perpendicular to each other $\left(\mathrm{AoT}=90^{\circ}\right)$ but never $180^{\circ}$ even at an inclination of $70^{\circ}$. This is of importance, since it underlines again the mismatched of real angle between impingement areas and current available hip cup design.

Present technical solutions of hip component design, which claims the prevention of dislocation and impingement, have not met desired demands. The approach of the bidirectional total hip prosthesis was adopted for a new implant design (Longevity Constrained Liner, Zimmer) (Figure 6). Based on our present data we predict a marked impingement with subsequent risk of dislocation due to leverage. Moreover, this implant does not have any sparing beneath the hemispheric rim. Instead, elevated rims are provided as a feature to avoid dislocation, which results in pseudo-gaps. Noteworthy, the pseudo-gaps are placed opposite to each other with an AoT of $180^{\circ}$ as well as areas with the elevated rim.

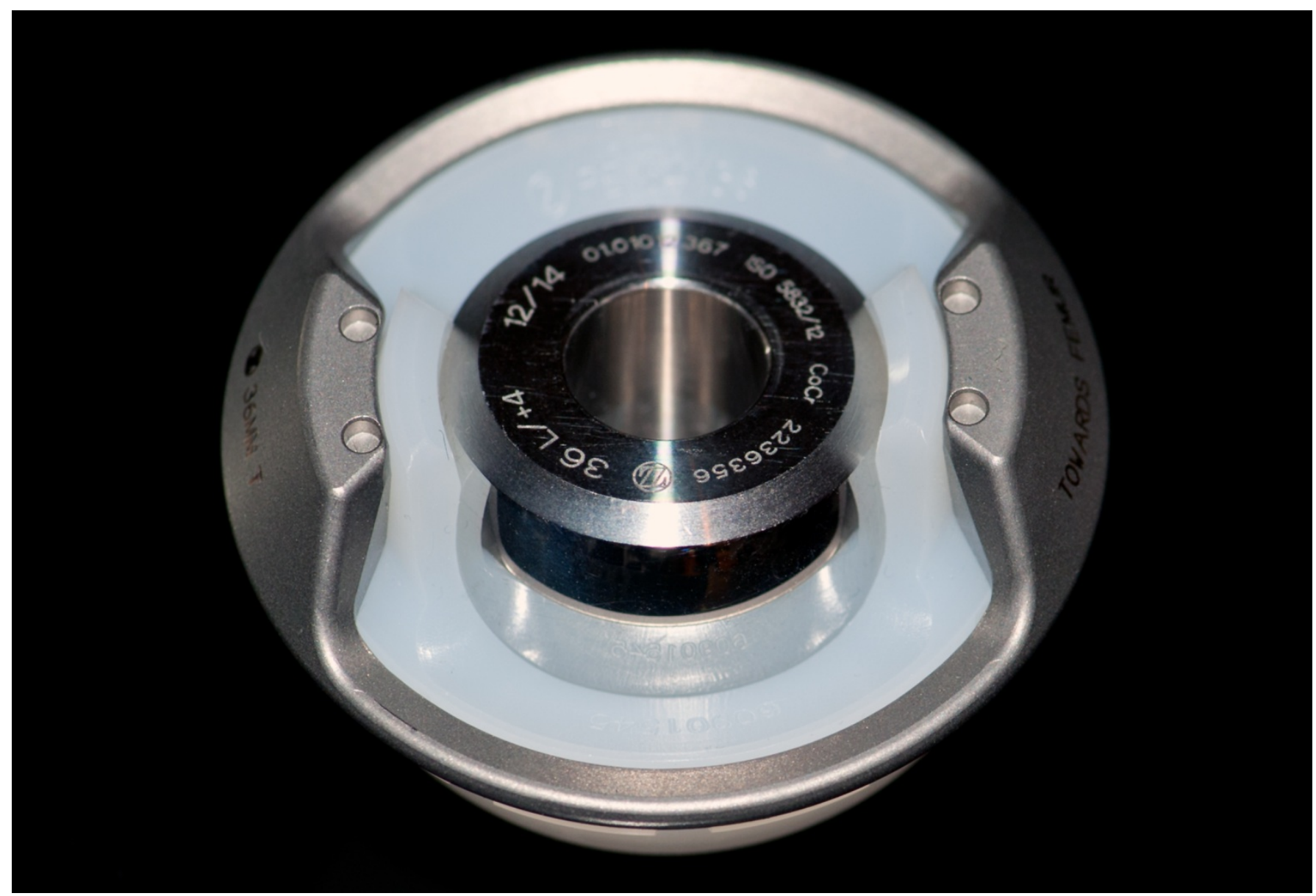

Figure 6: A commercial available constrained cup (Longevity ${ }^{\circ}$ Constrained Liner, Zimmer, Warsaw, Indiana, USA) with elevations and (pseudo) sparing gaps which are placed opposite to each other with an $\mathrm{AoT}$ of $180^{\circ}$. The present study clarified for the first time that the AoT with standard implant parameters is $108.3^{\circ}$ and far from the anticipated angle of $180^{\circ}$.

In addition, a rotationally symmetric elevated cup (snap fit, constrained cup) leads not only to possible psoas irritation, but also particularly to increased risk of impingement for which reason their application for revision THR is not recommended [14,15]. Based on our present data we suggest that the massive impingement, particularly in the areas of the anterior and posterior impingement is one fundamental reason for the failure of conventional constrained cups.

According to the operative instructions by the manufacturer, the rotational relationship should be provided so that the left hip implant is placed at approximately the one oclock position, and the right hip at approximately the eleven o'clock position. These values correspond to the findings of our study regarding the CoM but only for the anterior impingement point. Even though the rotation of a rotationally symmetrical design $\left(\mathrm{AoT}=180^{\circ}\right)$ is set on the anterior point of impingement, it will cause a massive limitation of RoM posteriorly because of the impact of the prosthetic neck on the elevated cup, and vice versa. 
Furthermore, even a rotationally symmetric cup reduction (reduced cup profile) increases the risk of dislocation as well as cup border pressure (increased abrasion). The use of larger heads primarily appeared reasonable due to reduced dislocation and impingement. However, an increased volumetric abrasion has been reported for larger heads, which resulted in a larger moment of friction [16]. In addition a 6.5 to 8.5 times higher plasma concentration of cobalt ions was reported for metal on metal pair when using $48 \mathrm{~mm}$ heads $(48 \mathrm{~mm}$ CormetTM Resurfacing und $46.8 \mathrm{~mm}$ Birmingham HipTM Resurfacing) in comparison to $28 \mathrm{~mm}$ heads (Metasul ${ }^{\circ}$ ) [17].

In contrast, the design of the bidirectional total hip prosthesis is not associated with a negative tribological effect as reported for larger hip head components given the bidirectional design solution is independent of the head diameter. By isolation of the two main trajectories of motion, the following advantages may be obtained without negative effects [1]:

- Avoidance of impingement

- Reduced dislocation

- Reduced cup border pressure

- Diminished psoas irritation

The design of a bidirectional total hip prosthesis demands a rotational asymmetry in order to prevent impingement but requires a specific rotation in the coronal cup plane during the implantation. The center of motion (CoM) is located at $22.0^{\circ}$ in the coronal cup plane and must be rotated externally to bring the spared cup regions into the desired positions. However, various simple implantation instruments as well as intraoperative movement checking maneuvers (e.g. clock model) are available which facilitate the technical obstacle.

Our present findings provide a basis for further development of implant design. From a theoretical point of view it would also be possible to create a hip cup with only one area of reduction and/or elevation. However, such a concept would dramatically change implantation parameters regarding positioning and alignment. We currently favor a mirrored symmetrical design (2 areas of sparing and/or elevations with an AoT $<180^{\circ}$ ), since surgeons can still use customary parameters for implantations with this design.

Further studies should consider how much further a design could be optimized to allow certain variability in implantation parameters including rotation. The main goal is an error-forgiving implant which covers the mobility requirements of active patients but also can be applied in a revision case [1].

\section{Conclusion}

Conventional implantation parameters yielded an implant design with rotational asymmetry $\left(\right.$ AoT $\left.<180^{\circ}\right)$ in the present RoM analysis. Complications such as dislocation and impingement may be avoided with this bidirectional total hip prosthesis. In addition, erosion caused by the tribological issues associated with larger head diameters and from expanded cup margins could be reduced. Moreover, the irritation of the psoas can be eliminated by reducing the anterior cup.

\section{Acknowledgement}

The authors would like to acknowledge Mr. Thomas Hermle (Aesculap AG, Research and Development, Tuttlingen, Germany) for providing the $\mathrm{CAD}$ images.

\section{References}

1. Nowakowski AM (2007) Luxation and Impingement nach Implantation von Hüfttotalprothesen, vermeidbare Komplikationen? Mechanischer Lösungsansatz für ein optimiertes Hüftgelenk-Endoprothesensystem: die bidirektionale Hüfttotalprothese. Orthop Prax 43: 348-355.

2. McCollum DE, Gray WJ (1990) Dislocation after total hip arthroplasty. Causes and prevention. Clin Orthop 159-170.

3. Soong M, Rubash HE, Macaulay W (2004) Dislocation after total hip arthroplasty. J Am Acad Orthop Surg 12: 314-321.

4. Burroughs BR, Hallstrom B, Golladay GJ (2005) Range of motion and stability in total hip arthroplasty with 28-, 32-, 38-, and 44-mm femoral head sizes. J Arthroplasty 20: 11-19.

5. Sanchez-Sotelo J, Haidukewych GJ, Boberg CJ (2006) Hospital cost of dislocation after primary total hip arthroplasty. J Bone Joint Surg Am 88: 290-294.

6. Phillips CB, Barrett JA, Losina E (2003) Incidence rates of dislocation, pulmonary embolism, and deep infection during the first six months after elective total hip replacement. J Bone Joint Surg Am 85: 20-26.

7. Bozic KJ, Kurtz SM, Lau E (2009) The epidemiology of revision total hip arthroplasty in the United States. J Bone Joint Surg Am 91: 128-133.

8. Shon WY, Baldini T, Peterson MG (2005) Impingement in total hip arthroplasty a study of retrieved acetabular components. J Arthroplasty 20: $427-435$.

9. Marchetti E, Krantz N, Berton C (2011) Component impingement in total hip arthroplasty: frequency and risk factors. A continuous retrieval analysis series of 416 cup. Orthop Traumatol Surg Res OTSR 97: 127-133.

10. Yamaguchi M, Akisue T, Bauer TW, Hashimoto Y (2000) The spatial location of impingement in total hip arthroplasty. J Arthroplasty 15: 305313.

11. Widmer $\mathrm{KH}$ (2007) Containment versus impingement: finding a compromise for cup placement in total hip arthroplasty. Int Orthop 31 Suppl 1:29-33.

12. Widmer KH, Majewski M (2005) The impact of the CCD-angle on range of motion and cup positioning in total hip arthroplasty. Clin Biomech Bristol Avon 20: 723-728.

13. Widmer KH, Zurfluh B (2004) Compliant positioning of total hip components for optimal range of motion. J Orthop Res Off Publ Orthop Res Soc 22: 815-821.

14. Noble PC, Durrani SK, Usrey MM (2012) Constrained cups appear incapable of meeting the demands of revision THA. Clin Orthop 470: 1907-1916.

15. Berend KR, Lombardi AV, Mallory TH (2005) The long-term outcome of 755 consecutive constrained acetabular components in total hip arthroplasty examining the successes and failures. J Arthroplasty 20: 93102.

16. Tarasevicius S, Kesteris U, Robertsson O, Wingstrand H (2006) Femoral head diameter affects the revision rate in total hip arthroplasty: an analysis of 1,720 hip replacements with 9-21 years of follow-up. Acta Orthop 77: 706-709.

17. Khan M, Takahashi T, Kuiper JH (2006) Current in vivo wear of metalon-metal bearings assessed by exercise-related rise in plasma cobalt level. J Orthop Res Off Publ Orthop Res Soc 24: 2029-2035. 